\title{
Mechanisms of Long-Term Potentiation: A Current-Source Density Analysis
}

\author{
Jeffrey S. Taube ${ }^{1, a}$ and Philip A. Schwartzkroin ${ }^{1,2}$ \\ Departments of ${ }^{1}$ Physiology and Biophysics and ${ }^{2}$ Neurological Surgery, University of Washington, Seattle, Washington \\ 98195
}

\begin{abstract}
A current-source density (CSD) analysis was carried out in the CA1 region of the hippocampal slice (1) to determine the pattern of current flow in pyramidal cells upon orthodromic stimulation and (2) to test the hypothesis that EPSPto-spike potentiation is produced by an alteration in this distribution of current sinks and/or sources. The results indicated that 2 sinks occur near the cell body layer (in addition to the sink associated with the EPSP) in response to orthodromic stimulation of the apical dendrites. An early (i.e., short-latency) sink was present along the radiatum/pyramidale border and was evident throughout the time course of the evoked field potential. This sink peaked in magnitude just prior to the peak of the population spike and was associated with orthodromic stimulation; it was not seen with antidromic stimulation. A second, later, sink occurred in the proximal portion of the basal dendrites and had a characteristic time course similar to the population spike; this second sink was also present during antidromic stimulation. There was some suggestion that the earlier dendritic sink shifted apically with development of long-term potentiation (LTP). The existence and movement of such an active zone in these cells may help to explain the dissociation of EPSP and spike potentiation in LTP.
\end{abstract}

An important, but poorly understood, finding from previous studies of long-term potentiation (LTP) mechanisms (Andersen et al., 1980, 1984) is that cell propensity for spike initiation in response to orthodromic stimulation increases following highfrequency stimulation (HFS), a change often seen despite an unchanged EPSP (Bliss and Lomo, 1973; Taube and Schwartzkroin, 1988). Several investigations (Andersen et al., 1980; Wilson, 1981; Wilson et al., 1981; Abraham et al., 1985) have indicated that the population spike is potentiated more than can be explained simply on the basis of potentiation of the population EPSP. These findings suggest a change in the coupling between EPSP and spike initiation.

The existence of an EPSP-to-spike (E-S) component in LTP

\footnotetext{
Received Sept. 22, 1986; revised Sept. 21, 1987; accepted Sept. 23, 1987.

We wish to thank Carol Robbins for assistance in preparing some of the figures. This research was supported by NIH Grants NS 18895 and GM 07108, and NSF Grant BNS 8209906. P.A.S. is an affiliate of the Child Development and Mental Retardation Center, University of Washington.

Correspondence should be addressed to Jeffrey S. Taube, SUNY-Health Sciences Center, Department of Physiology, Box 31, 450 Clarkson Avenue, Brooklyn, NY 11203

a Present address: Department of Physiology, SUNY-Health Sciences Center, Brooklyn, NY 11203.

Copyright (C) 1988 Society for Neuroscience $0270-6474 / 88 / 051645-11 \$ 02.00 / 0$
}

argues for mechanisms other than facilitation of synaptic transmission as critical to LTP. One possibility, yet unexplored, is that the E-S potentiation is produced by some alteration in the distribution of current sinks and/or sources in the neurons. For example, changes in the density and/or location of dendritic calcium and sodium channels (Wong et al., 1979; Benardo et al., 1982) could lead to altered patterns of current flow in the dendrites. A change in the origin or intensity of current flow in the dendrites could alter transfer of current to the spike initiation zone. To test this hypothesis, we performed a one-dimensional current-source density (CSD) analysis before and after a LTP conditioning train.

Preliminary reports concerning some of these findings have been presented (Taube and Schwartzkroin, 1985).

\section{Materials and Methods}

Transverse slices from adult guinea pig hippocampus were prepared and maintained as described in the accompanying paper (Taube and Schwartzkroin, 1988). One-dimensional CSDs are often calculated by using averaged field potentials from a single, but moving, microelectrode. This calculation uses the average of several events over time; consequently, small changes in the field potential from one event to the next may be obscured in the averaging process. In order to avoid this potential problem, we constructed a 3-electrode array using a Narshige triple manipulator. The array was moved along a track parallel to the axis of the hippocampal pyramidal cells. This procedure enabled us to record and calculate the CSD at a specific site for a single event.

To construct the array, 3 extracellular microelectrodes were madc from fiber-filled glass micropipettes and filled with $2 \mathrm{M} \mathrm{NaCl}$ (tip resistance was approximately 5-10 $\mathrm{M} \Omega$ ); each of the microelectrodes had a similar lip resistance $( \pm 2 \mathrm{M} \Omega$ ). The 3 electrodes were spaced $25-30 \mu \mathrm{m}$ apart and aligned perpendicular to the $C A 1$ pyramidal cell layer. The triple-electrode array was moved along a track perpendicular to stratum (str.) pyramidale, at intervals of $30 \mu \mathrm{m}$, from str. radiatum to str. oriens (Fig. 1). Accurate placement of the array was accomplished with the aid of a graticule placed in the eyepiece of a stereomicroscope. At each position, the array was lowered into the slice to a depth of $50 \mu \mathrm{m}$; deeper penetrations were avoided in order to prevent possible tissue injury.

A special pair of bipolar stimulating electrodes was constructed such that the tips were fixed $70-80 \mu \mathrm{m}$ apart. The stimulating electrodes were placed in the str. radiatum at a distance of $200-300 \mu \mathrm{m}$ from the str. pyramidale and were positioned along an axis parallel to the str. pyramidale in an attempt to activate a discrete fiber bundle. Prior to HFS, evoked field potentials were collected for 3 or 4 different stimulus strengths. The lowest stimulus intensity was adjusted until a small (usually $1-2 \mathrm{mV}$ ) population spike was present in the field potentials. Baseline stimulation frequency for the CSD experiments was $0.2 \mathrm{~Hz}$. At each location of the triple-electrode array, 6 field responses were recorded and averaged for each stimulus strength. Following collection of field responses, the triple-electrode assembly was returned to the pyramidal cell body layer. Subsequently, the magnitude of the evoked population spike was compared with the magnitude of the population spike evoked from the same location at a time prior to collection of field responses. If the magnitude of the 2 population spikes were within $10 \%$ of one 


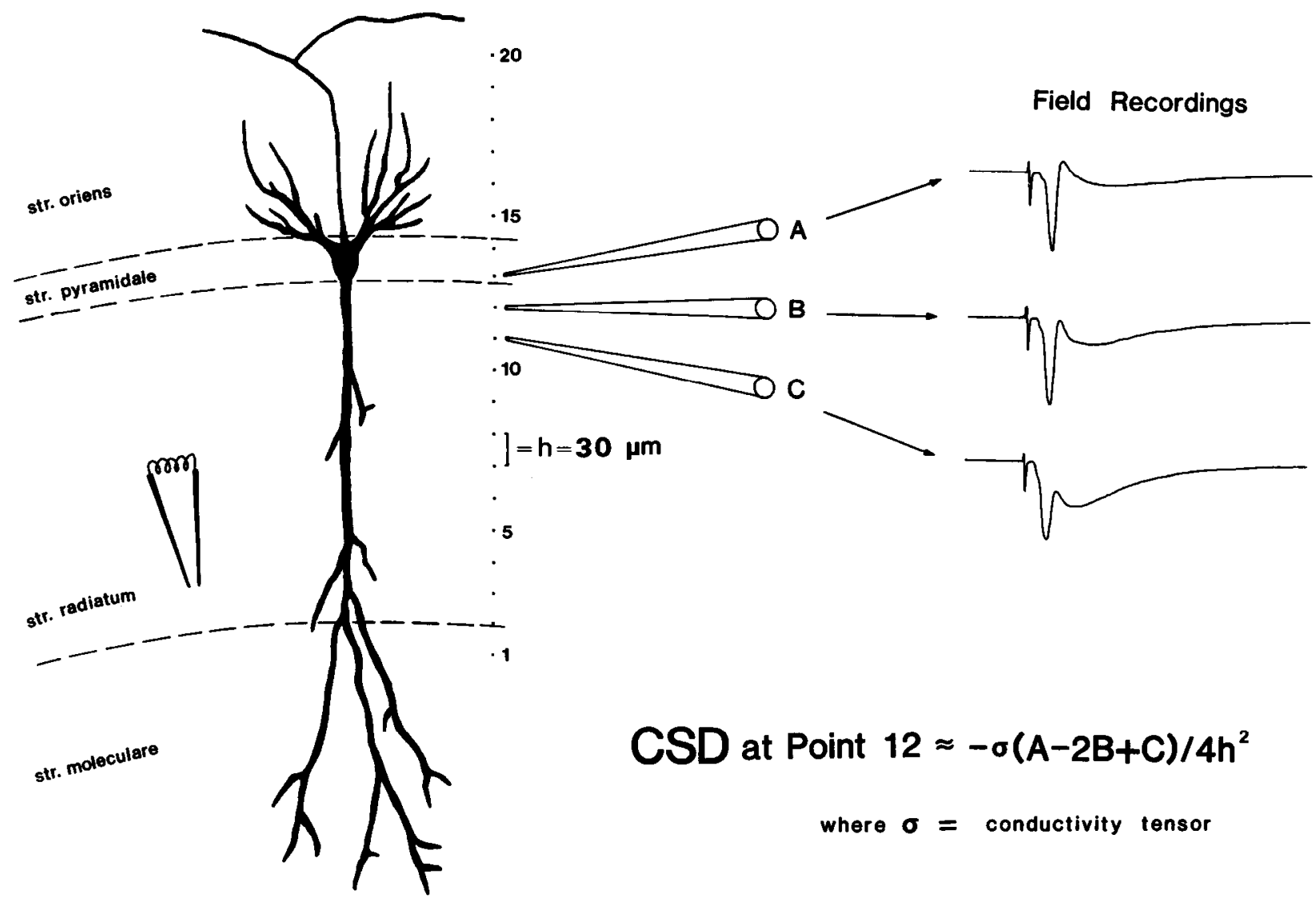

Figure 1. Illustration of the procedure used for the triple-electrode array CSD measurements. Three electrodes $(A-C)$, spaced $30 \mu \mathrm{m}$ apart, were aligned perpendicular to the stratum (str.) pyramidale in CA1 hippocampus. The 3 electrodes were moved along a track parallel to the pyramidal cell axis, at $30 \mu \mathrm{m}$ intervals, beginning at position 1 and ending at position 20 . At each location, several field potential responses were recorded in response to stimulation of str. radiatum $($ Stim $) ; 3$ different stimulus strengths were tested. Six CSD records were calculated from 6 field potential responses at each stimulus strength, and these CSDs were averaged. In addition, the 6 field potential responses from the middle electrode $(B)$, at each different stimulus strength, were averaged (shown on the right). Two trains of HFS (100 Hz, $1 \mathrm{sec})$, spaced 3 sec apart were applied to str. radiatum at the low stimulus strength. If, after $20 \mathrm{~min}$, LTP had developed in the population spike, another series of 6 field potential responses were obtained at the low stimulus strength condition.

another, then $2 \mathrm{HFS}$ trains $(100 \mathrm{~Hz}, 1 \mathrm{sec}$ each), spaced $3 \mathrm{sec}$ apart, were delivered through the same stimulation electrode at the low stimulus strength. Field responses were again recorded at the baseline stimulation rate, starting at 20 min after the HFS.

The amplified voltage signals were stored on magnetic tape. The CSDs were calculated and analyzed off-line on a Norland 3001 computer. Population spike amplitude was computed as the difference between the peak negativity and the averaged value of the preceding and following peak positivities. LTP was defined as being present when the average population spike amplitude rccorded at the cell body layer $20 \mathrm{~min}$ following HFS was at least $125 \%$ of control values.

The one-dimensional CSD at point $x$ was calculated using the following formula:

$$
I_{x}=-\sigma\left(E_{x-h}-2 E_{x}+E_{x+h}\right) / 4 h^{2}
$$

where $I_{x}=$ the current at location $x, h=$ the sampling distance $(h=30$ $\mu \mathrm{m}$ in our experiments), $E_{x}=$ the extracellular voltage at location $x$, $E_{x-h}=$ the extracellular voltage at location $x-h, E_{x+h}=$ the extracellular voltage at location $x+h$, and $\sigma=$ the tissue conductivity tensor.

Reported values for the tissue conductivity vary for different neuroanatomical regions of the brain (Ranck, 1963; Nicholson and Freeman, 1975.). The value is dependent on the direction of current flow and the anatomical barriers present within the tissue. Because of the anisotropic nature of the nervous system, assigning a precise value for $\sigma$ in our calculations is difficult and subject to error. Since the purpose of our study was to make a comparison between the relative CSD values before and after development of LTP, we have chosen not to include this term in our calculations; instead, we have expressed the CSD as
$\mathrm{mV} / \mathrm{cm}^{2}$. Thus, our computed values are only proportional to the true CSD.

\section{Results}

Triple-electrode array experiments

Nine experiments with the triple-electrode array were carried out in slices showing LTP of the population spike $20 \mathrm{~min}$ following HFS. Prior to HFS, CSD records were collected for 3 or 4 different stimulus strengths. The lowest stimulus intensity was adjusted to evoke a small population spike $(1-2 \mathrm{mV})$ in str. pyramidale, and this stimulus strength was used for HFS. If the population spike (measured in str. pyramidale) was potentiated 20 min following HFS, another track of field potentials (evoked at the low stimulus strength) was recorded and the CSD records computed. The particular pre-HFS stimulus strength that produced a pyramidale population spike most similar to the postHFS population spike was used for comparing differences in the CSD records. The sink associated with pyramidal cell discharge per se was thus "equated," so that differences between pre- and post-HFS CSDs could be attributed to an EPSP-to-spike coupling mechanism. An estimate of the "closeness" between the 2 experimental conditions being compared was calculatcd by taking the ratio between the peak amplitude of the population 
spike at the high stimulus strength pre-HFS condition and the peak amplitude of the population spike at the low stimulus strength post-HFS condition. In the 9 experiments described below, the mean ratio between the magnitudes of these 2 population spikes was $0.974 \pm 0.322$. The CSD records were quite noisy and were therefore computer-smoothed ( 5 point averaging). Six "smoothed" records were collected at each electrode location for each stimulus strength used; these records were then averaged for analysis.

The first columns of Figure $2 A-C$ depict the averaged extracellular voltage recordings from one experiment under 3 different stimulus conditions. The voltage traces represent the averaged extracellular recordings obtained from the middle electrode of the triple-electrode array. The conditions shown are (1) pre-HFS at low stimulus strength, (2) pre-HFS at high stimulus strength (i.e., a stimulus intensity producing a response similar to 3), and (3) post-HFS at low stimulus strength. The average of 6 computed CSDs for each electrode position is illustrated in the second columns of Figure $2 A-C$. To help clarify the differences between the pre- and post-HFS CSD records, 6 time points were chosen for analysis and the CSD value at each time point measured. The 6 time points, shown in the lowerleft portions of Figures 3 and 4, were chosen based on the extracellular voltage waveform evoked in str. pyramidale, before HFS, at the stimulus strength that produced a field recording comparable to the post-HFS potentiated response. The time points were as follows:

Time point a: midway up the initial rising slope.

Time point $b$ : peak of the first positivity.

Time point $\mathrm{c}$ : midway down the initial negative-going slope.

Time point $\mathrm{d}$ : peak of the negativity.

Time point e: midway up the second rising slope.

Time point $f$ : peak of the second positivity.

The CSD value for each time point was then replotted versus its position along the pyramidal neuron (Fig. 3). The results from averaging CSDs from 9 different experiments are shown in Figure 4.

Prior to HFS, orthodromic activation of the apical dendrites in the distal portion of str. radiatum produced 2 different and distinct sinks near str. pyramidale (Figs. 3, 4). At a time before the first peak positivity of the field potential (time point a), a weak sink (referred to as the "early sink") occurs at the pyramidale/radiatum border and a broader, weak source (referred to as the "early source") occurs along the pyramidale/oriens border. At the peak of the first field positivity (time point b), the early sink and source both increase in magnitude. A spatially widespread, weak source is present throughout the proximal portion of str. radiatum at time point a and remains through the first peak positivity in the field recording (time point b); it wanes during the downward phase of the population spike (time points $\mathrm{c}$ and $\mathrm{d}$ ) and is no longer seen during and after the upward (positive-going) phase of the population spike (time points e and f). During the downward phase of the population spike (time point c), the early sink reaches its peak amplitude and occurs over a larger portion of the proximal apical dendrites. In addition, a second sink (referred to as the "late sink") begins to develop in str. oriens, about $60 \mu \mathrm{m}$ from the cell body layer. At time point $c$, the early source magnitude is increased compared with time point $b$. Furthermore, a second source develops (referred to as the "late source") in the distal portion of str. oriens, particularly at the higher stimulus strength condition and in the
post-HFS record. At the peak of the population spike (time point d), the early sink is decreased; the late sink attains its largest amplitude under the high stimulus strength and post-HFS conditions but is absent during the low stimulus strength condition. Both the early and late sources are present at time point $\mathrm{d}$, with the late source remaining very small in the low stimulus strength condition. During the upward phase of the population spike (time point e), the early and late sinks decline in magnitude; the early source remains prominent, but the late source amplitude is much reduced. At the peak of the second positivity in the field recording (time point $f$ ), the early sink and early source are still present while the late sink and late source have disappeared.

Our CSD records do not indicate a clear sink in the apical dendrites which reflects local synaptic activation. The likely explanation for this absence is that our bipolar stimulating electrodes, even when optimally positioned, activated a relatively wide band of fibers in the apical dendritic region. Our sampling interval of $30 \mu \mathrm{m}$ was therefore too small to detect a discrete synaptic sink. To check for this possibility, we computed the CSD values using "the second nearest neighbor" technique, with a sampling interval of $60 \mu \mathrm{m}$. With this procedure, an apical dendritic sink corresponding spatially to the level of placement of the stimulating electrode became evident (see CSD values for time points $c$ and $d$ in Fig. 6).

Following HFS, the pattern of sinks and sources did not change dramatically. However, in 3/9 experiments there was a small shift in the early sink toward the apical dendrites (indicated by an asterisk at time points $c$ and $d$ in Figs. 3 and 4). The shift was approximately one recording point, a distance of $30 \mu \mathrm{m}$. Figures 2 and 3 depict the results from 1 of these 3 experiments. In this experiment, the shift was most prominent during the development and peak of the population spike (time points bd). When the sink shifted, the early source shifted in parallel, most prominently during the peak of the population spike (time point d). In addition, the late sink and source appeared to broaden toward the cell body layer. In 2 of the remaining 6 cxperiments, a $30 \mu \mathrm{m}$ shift in the early sink could be detected, but the shift was subtle. The remaining 4 experiments showed no movement of sink or source from pre- to post-HFS conditions. The presence or absence of a shift in the early sink following HFS was not a function of the amount of potentiation in the population EPSP or population spike. In 2/4 experiments where there was no shift in the early sink, there was a high degree of potentiation in the population EPSP and population spike.

The averaged CSD records (Fig. 4) indicate small shifts in the early sink and early source but not in the late sink and late source. Statistical tests between the pre-HFS high stimulus strength condition and the post-HFS low stimulus strength condition indicate a significant difference in the early sink magnitude during the downward phase and at the peak of the population spike (time points $\mathrm{c}$ and $\mathrm{d} ; p<0.05$, Student's $t$ test).

In 2 of the 9 experiments, we computed the CSDs using both the triple-electrode array and the average field potential obtained from the middle electrode of the electrode array. The comparison showed no significant differences between techniques. There was, however, more noise and variability in the procedure using the field potentials averaged from the single electrode. Since the small sink shifts seen in the triple-electrode array experiments were not consistently observed, we attempled to clarify this inconsistency by performing 2 other types of experiments. First, we calculated CSDs in the CAl area evoked by antidromic stimulation. Second, we conducted a CSD anal- 


\section{Low Stimulus Intensity}

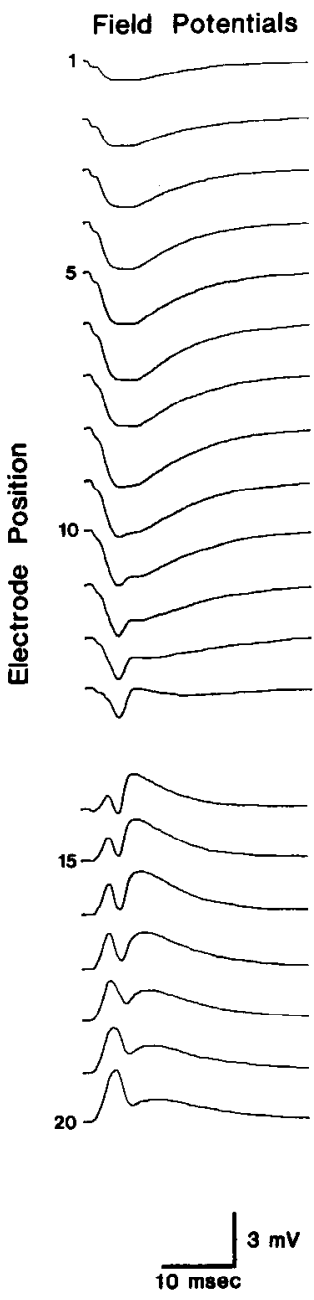

CSD Records

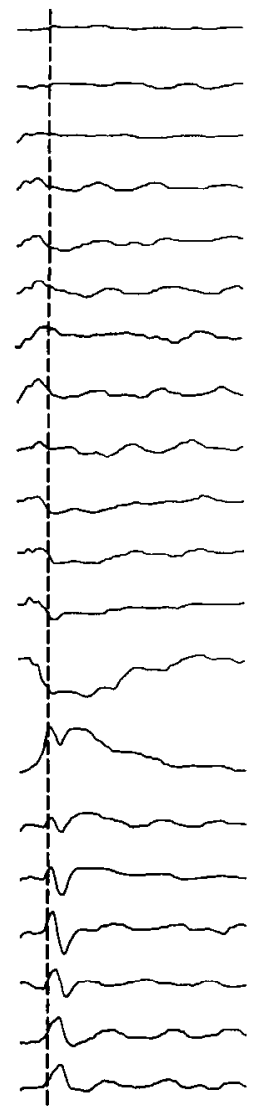

$\overbrace{\text { Sink }}^{\text {Source }} 400 \mathrm{mV} / \mathrm{mm}^{2}$
High Stimulus Intensity

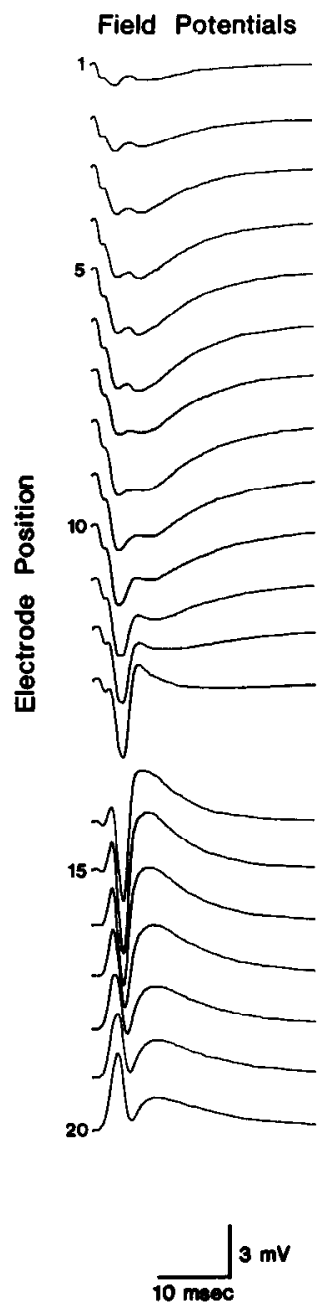

CSD Records

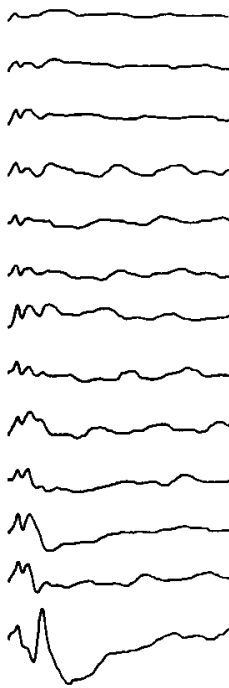

$\sqrt{ }$
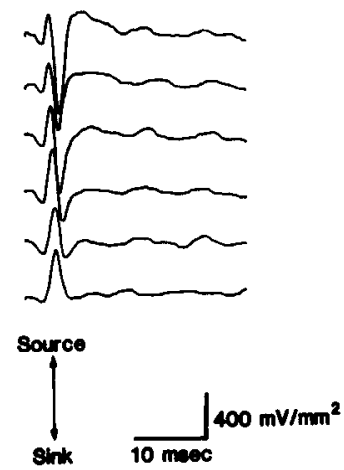

Figure 2. Field potentials (left columns) and calculated CSD records (right columns) in response to orthodromic stimulation under 3 different stimulus conditions. The position number of the middle electrode of the triple-electrode array is shown on the left; position 1 corresponds to str. radiatum/moleculare border and position 20 to the str. oriens/alveus border (see Fig. 1). The cell body layer is located between positions 13 and 14. Orthodromic stimulation was applied to the distal str. radiatum. $A$, Low-intensity stimulus strength; $B$, high-intensity stimulus strength; $C$, post-HFS. Responses were obtained at the low stimulus strength, 20 min after delivery of 2 trains of HFS to str. radiatum. Each field potential and CSD record is an average of 6 traces. Positivity (sources) is upward; negativity (sinks), downward. The dashed line running through the CSD records in $A$ illustrates where CSD values for time point c were chosen for constructing the graphs in Figure 3.

ysis when 2 different orthodromic pathways were activated but only one pathway received HFS. These experiments were performed with a single electrode recording procedure, rather than with the triple-electrode array.

\section{Antidromic experiments}

In 4 antidromic stimulation experiments, 2 bipolar stimulating electrodes were used, one positioned in the alveus and the second in the distal portion of str. radiatum (S1 and S3 in Fig. 5). A series of pre-HFS field recordings were collected for both orthodromic and antidromic stimulation; a similar series was collected 20 min following a LTP-inducing train of HFS applied to the orthodromic pathway. The CSDs were computed as in the previous experiments and CSD graphs constructed based on the 6 different time points. An additional 3 time points were chosen for the antidromic stimulus based on the pre-HFS antidromically evoked waveform in str. pyramidale. The first time point (time point $\mathrm{x}$ in the lower-right corner of Fig. 6) corresponded to the midpoint on the initial declining (negativegoing) slope; the peak of the negativity was chosen for the second time point (time point $y$ in Fig. 6); and the third time point corresponded to the midpoint on the following rising (positivegoing) slope of the antidromic field potential (time point $\mathrm{z}$ in Fig. 6).

As in the triple-electrode array, experimental results indicated the presence of 2 distinct sinks with orthodromic stimulation. However, antidromic stimulation produced a sink only near the pyramidale/oriens border. This observation suggests that the sink near the pyramidale/oriens border can be attributed to activation of the initial segment "trigger" region of a group of pyramidal cells. In these experiments, no movement of the sinks and sources, produced by orthodromic stimulation, was observed following a period of HFS to the orthodromic pathway (Fig. 6, time points $\mathrm{c}$ and $\mathrm{d}$ ). However, post-HFS CSD analysis 


\section{Post-HFS}

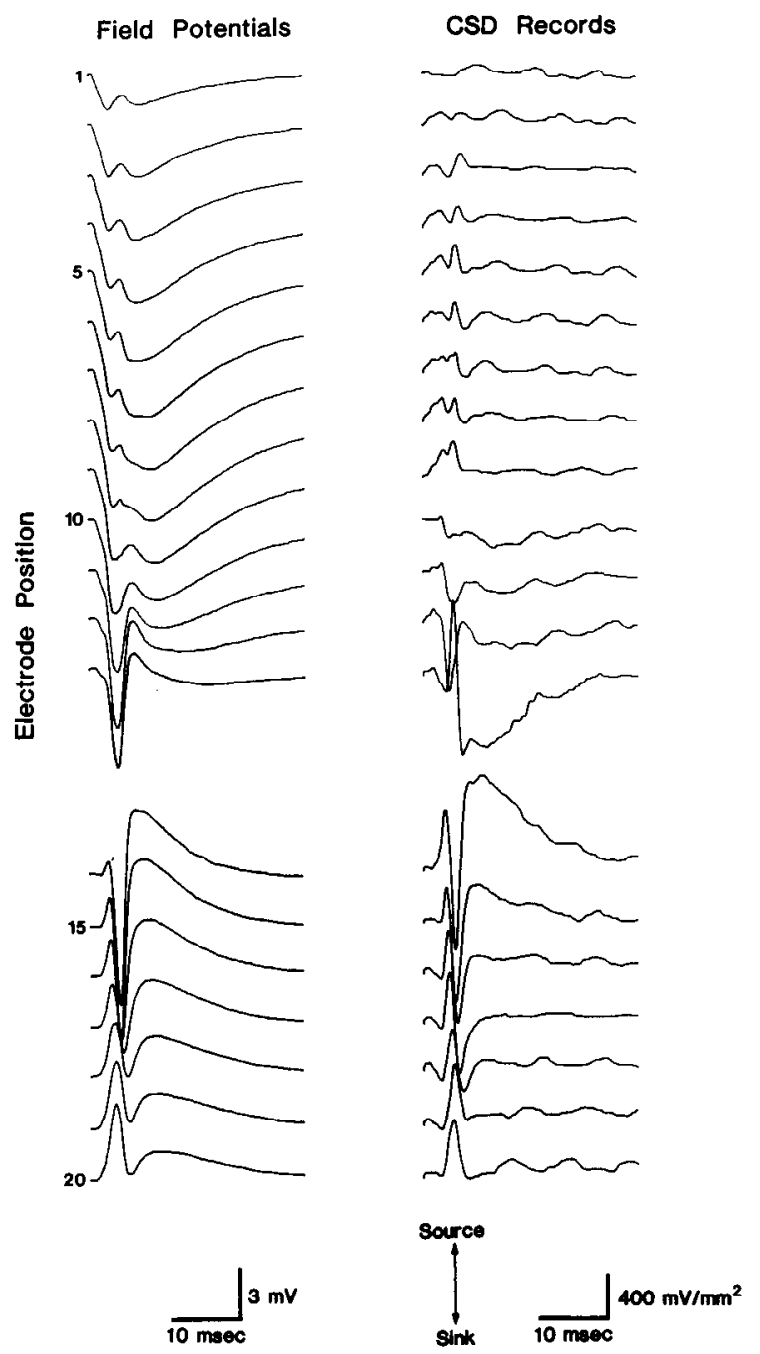

of the antidromically elicited potential showed a $30 \mu \mathrm{m}$ shift of the sink toward the basal dendrites in $2 / 4$ experiments.

\section{Double orthodromic inputs}

In the double orthodromic input experiments, 2 stimulating electrodes were positioned in different portions of str. radiatum. One electrode (S1 in Fig. 5) was located as in previous experiments, near the radiatum/moleculare border. The second electrode (S2 in Fig. 5) was positioned in the proximal portion of str. radiatum, on the "opposite side" of the recording electrode track from the first stimulating electrode. For these studies, it was critical that the 2 stimulating electrodes activated different groups of fibers. We used a paired-pulse manipulation (with the 2 orthodromic inputs separated temporally by $50 \mathrm{msec}$ ) to test this independence. Stimulating electrode placements were considered acceptable when a "test" pulse delivered to electrode S1 showed no population spike facilitation by a conditioning pulse delivered to elcctrode S2 (Fig. 7B). Homosynaptic paircdpulse testing (i.e., paired-pulse testing with a given stimulating electrode) always produced a significant increase in the test population spike as compared to the conditioning population spike
(Fig. 7C). This method has been used previously for selective activation of different orthodromic inputs (Creager et al., 1980).

As with the antidromic experiments, only a single recording electrode was used for CSD analysis. Five extracellular field potential samples were collected and averaged at each location along the recording track. In 3 separate experiments, pre-HFS CSD records indicated the presence of 2 different sinks for both orthodromic inputs. LTP was observed only in the pathway receiving the HFS; the nontetanized pathway was not potentiated, confirming earlier reports of the homosynaptic nature of LTP in the CA1 area (Schwartzkroin and Wester, 1975; Andersen et al., 1977, 1980; McNaughton and Barnes, 1977; Alger et al., 1978). CSD analyses indicated no movement of either the early or late sink, in either tetanized and untetanized pathways, following induction of LTP.

\section{Discussion}

The purpose of the CSD experiments were 3-fold: first, to determine the pattern of current flow in pyramidal cells upon orthodromic activation; second, to determine whether this pattern changed following development of LTP; and third, if the sink/source pattern was altered, to determine if the altered pattern could explain E-S potentiation.

Current-source density analysis utilizes extracellular recordings to determine the temporal and spatial distributions of current sinks and sources in nerve tissue. Nicholson and Freeman, applying current-source density theory to nervous system tissue, described a 3-dimensional mathematical model to be used for calculating the CSD (Freeman and Nicholson, 1975; Nicholson and Freeman, 1975). The accuracy of applying this technique along a single axis (i.e., one-dimension) was also studied using a cylindrical model. They demonstrated that application of the one-dimensional CSD analysis was warranted only when the ratio of diameter to length of the cylinder had values $>1$. A large synchronous ensemble of neurons was also necessary to insure accuracy. In the present experiment, small interelectrode sampling distances (yielding a diameter-to-length ratio $>1$ ), coupled with the synchronous response of pyramidal neurons to stimulation, satisfied these requirements for applying the onedimensional CSD technique.

In applying the CSD technique to the hippocampal slice CA1 region, 2 key assumptions must be considered. First, it is assumed that the conductivity of the extracellular tissue does not change following induction of LTP. In addressing this issue, Dietzel et al. (1982) found no significant changes in extracellular volume (and by extrapolation, in extracellular conductivity) following conditioning trains. Second, the CSDs were computed with the assumption that the extracellular tissue in the CA1 region was isotropic. We have no data to support this contention. In fact, Jefferys (1984) has found that the extracellular resistivity in the pyramidal and granule cell body layers is 1.5 3 times that in dendritic layers. When this increased resistivity is taken into account in our calculations, the intensity of the sinks and sources are reduced slightly in the pyramidal cell body layer, but the overall pattern and location of the sinks and sources remain unchanged.

Our CSD experiments revealed 2 spatially distinct sinks near the cell body layer (and distinct from the sink corresponding to the dendritic EPSP). The first of these sinks (the "early" sink) peaked just prior to the peak of the population spike and was localized near the pyramidale/radiatum border. The second sink (the "late" sink) developed coincident with the peak of the pop- 
Figure 3. Comparison of CSD amplitudes at 6 different time points for 3 different stimulus conditions. Six time points $(a-f)$, which represent identified components of the pre-HFS orthodromically evoked waveform recorded at the str. pyramidale/oricns bordcr (sce inset in the lower-left corner) were chosen for analysis. CSD amplitudes were measured at these 6 time points for each position of the triple-electrode array. Plots were then constructed comparing the CSD amplitudes (ordinate), under the 3 different stimulus conditions, relative to the electrode position along the pyramidal neuron axis (abscissa). The 3 different stimulus conditions were: low stimulus strength, high stimulus strength, and low stimulus strength, 20 min after 2 trains of HFS $(100 \mathrm{~Hz}, 1$ sec) spaced 3 sec apart. The CSD amplitude graphs indicate the development of 2 distinct sinks near the cell body region. The early sink appeared at time point a, peaked at time point $c$, and was still present at time point $f$. The late sink, which was evident only under conditions of high stimulus strength and post-HFS conditions (i.e., when there was a large population spike), first appeared at time point $c$, peaked at time point $d$, and was no longer present by time point $f$. A well-defined shift in the early sink (asterisk) occurred at time points $c$ and $d$ in the post-HFS records from this slice. The flat portion of the plot (left and right extremities of each trace) represents zero (i.e., no current flux).
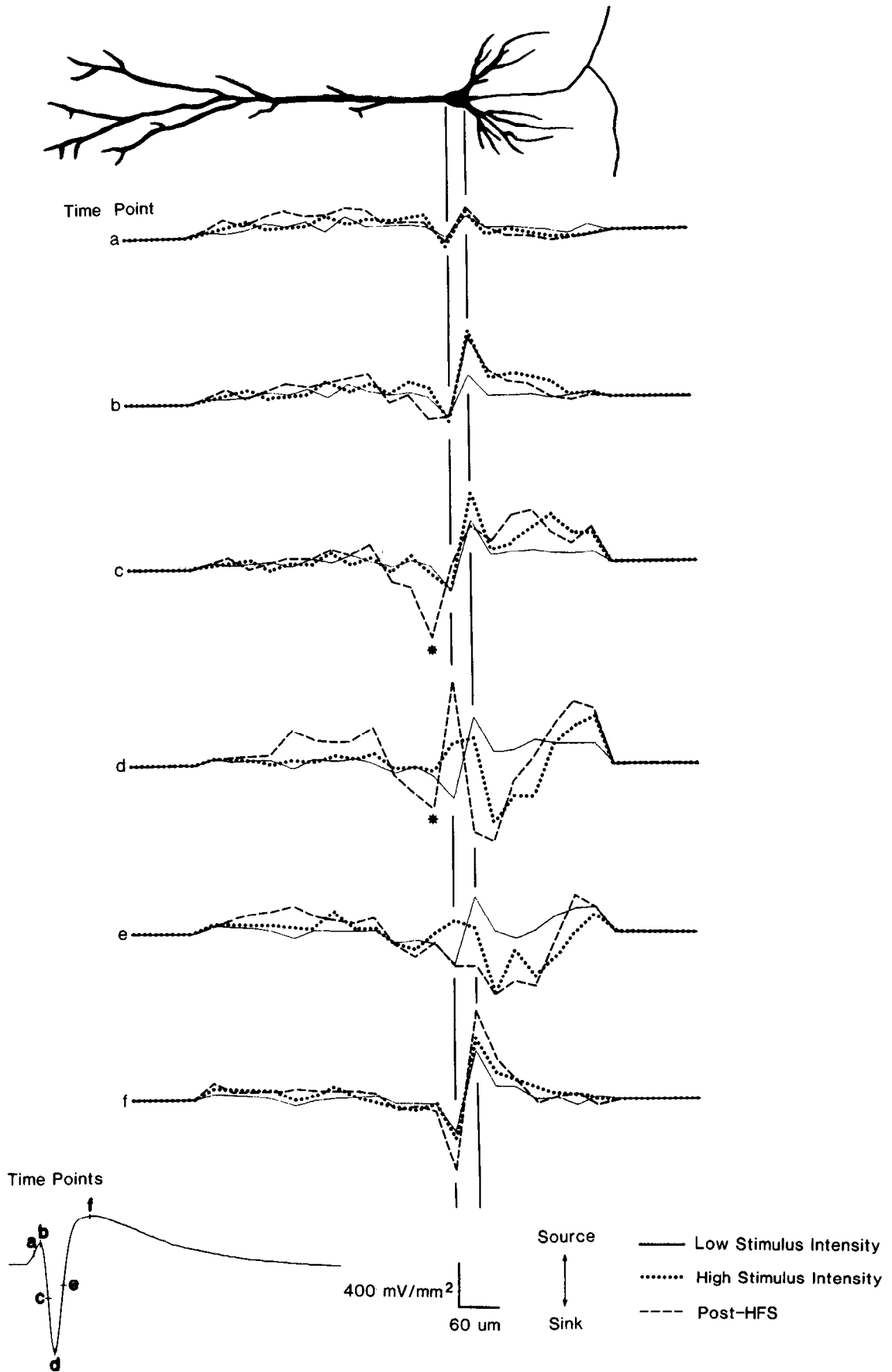

ulation spike and was localized to str. oriens, 30-60 $\mu \mathrm{m}$ from the cell body layer. The EPSP sink could be detected in the distal portion of str. radiatum (when the second-nearest-neighbor procedure was used to compute the CSDs and was broadly distributed.
Generation of the late sink seems clear-cut. The late sink first appears when the population spike is initiated (time point $c$ ), reaches a peak at the time of the peak in the population spike (time point d), and disappears when the population spike has ended (after time point e). When the stimulation intensity was 


\section{Comparison of CSD Amplitudes at 6 Different Time Points Averaged Across 9 Animals}

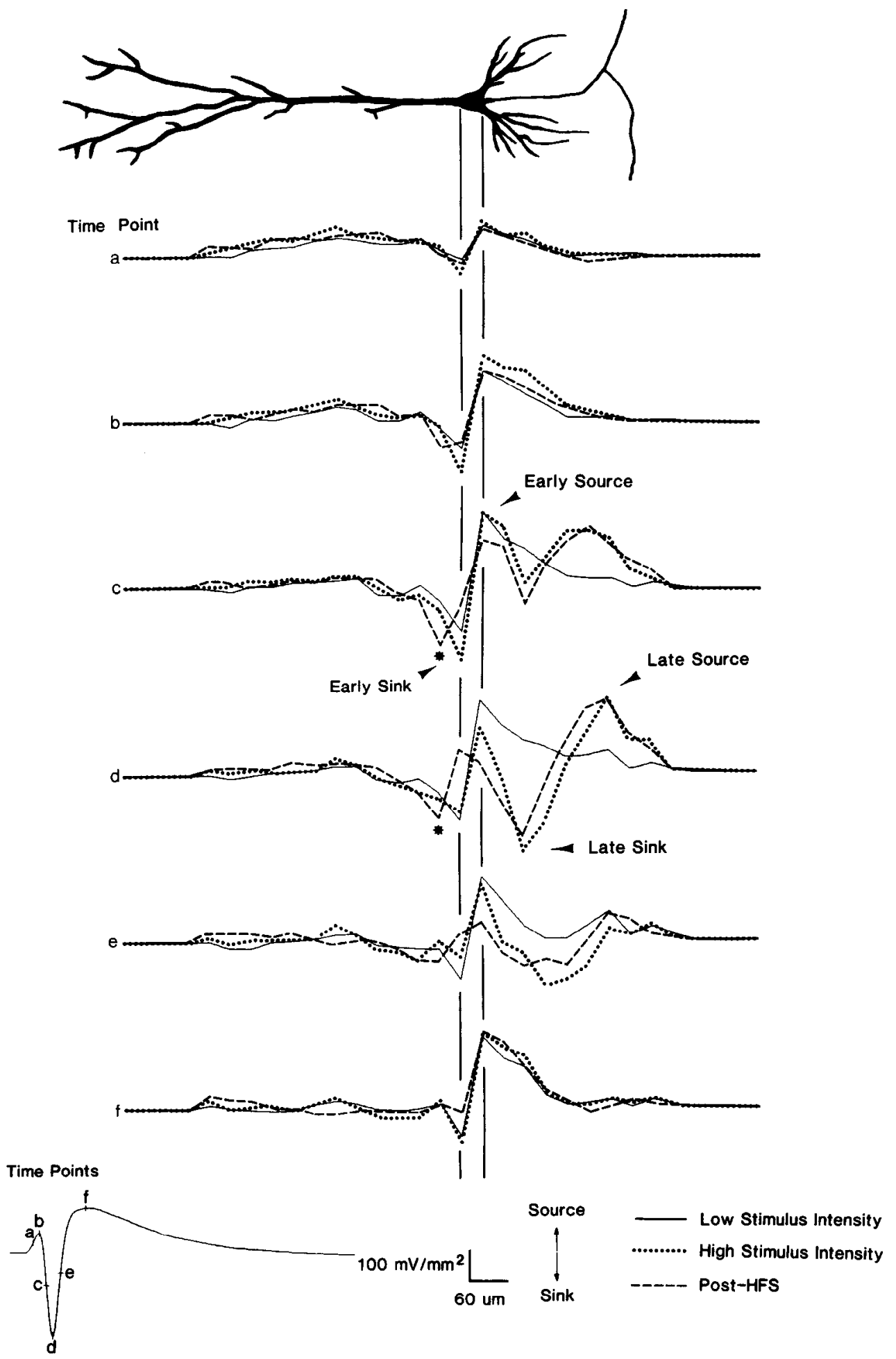

Figure 4. Graph similar to Figure 3, except each plot represents the average CSD profile from 9 different slices/experiments. The early sink and source are labeled in trace (time point) $\mathrm{c}$; the late sink and source are labeled in trace d. The post-HFS shift in the early sink (asterisk) was significant in traces $\mathrm{c}$ and $\mathrm{d}(p<0.05)$. subthreshold for population spike generation, the late sink did not appear in the CSD records. CSD records computed during antidromic stimulation indicate the presence of only this sink (not of the early sink). These observations indicate that the late sink is associated with the discharge of pyramidal neurons; the sink appears in proximal str. oriens because the initial segment trigger zone of the pyramidal neurons is located there.

The development of a sink at the radiatum/pyramidale border is less easily explained. This sink develops very early in timeit was evident at the first measured time point-and increased 


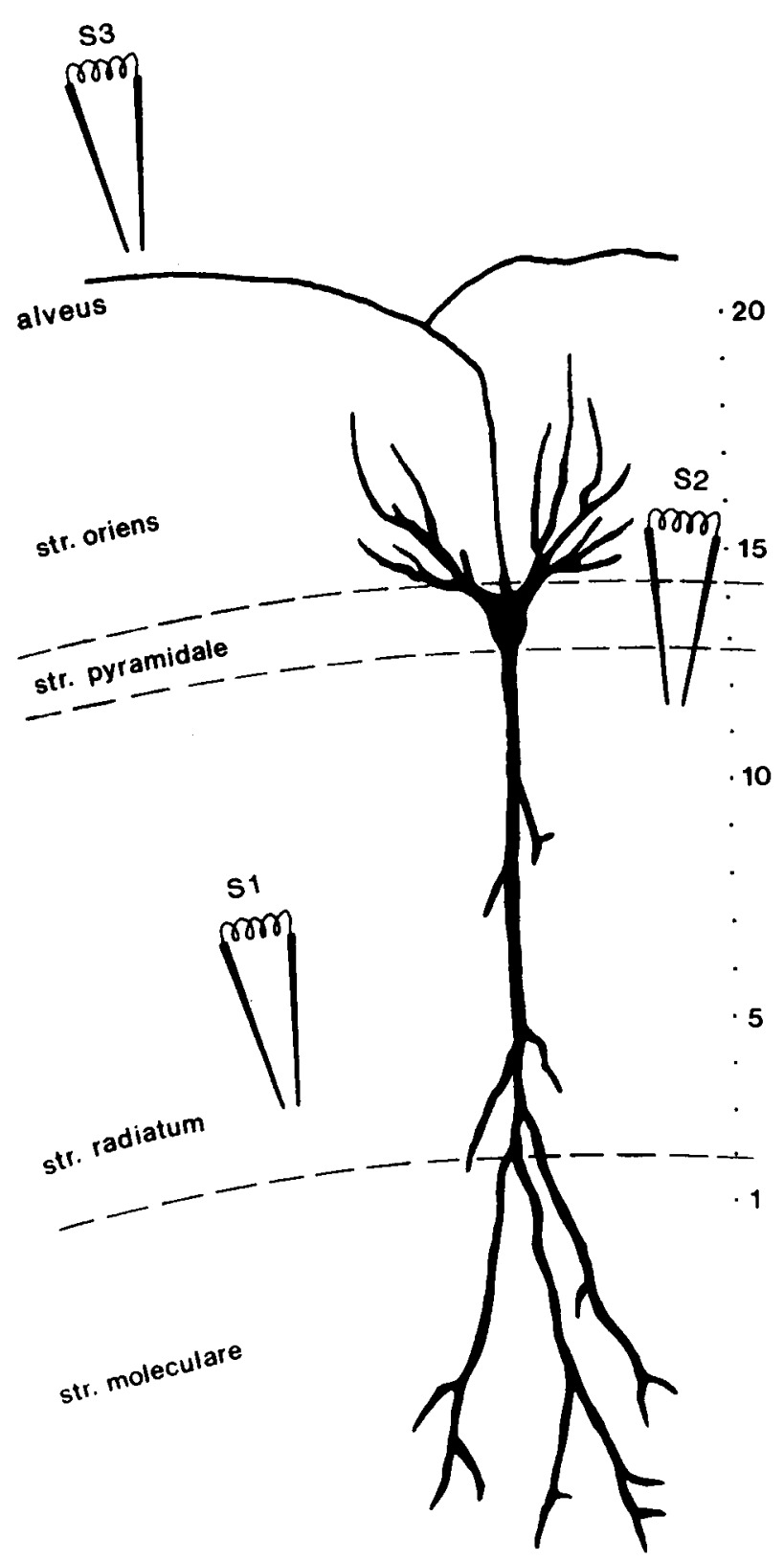

Figure 5. Illustration of the position of the stimulating electrodes in the CAl area for the antidromic and double orthodromic stimulation experiments. For the antidromic experiments, stimulating electrodes were positioned at S1 and S3. For the double orthodromic experiments, stimulating electrodes were positioned at $\mathbf{S} 1$ and $\mathrm{S} 2$.

to its peak amplitude just prior to the peak of the population spike. The graphs of the computed CSD averages indicate that this sink has a prominent spatial distribution along the radiatum/pyramidale border and spreads into the apical dendrites for $70-80 \mu \mathrm{m}$. This sink cannot be attributed to the discharge of pyramidal neurons because it (1) occurs before the population spike, (2) is absent from the antidromically activated system, and (3) occurs under conditions of low stimulus intensity when pyramidal cell discharge is minimal. This sink presumably represents the activation of channels in the very proximal portion of the pyramidal cell apical dendrites. Existence of active sodium and calcium channels in pyramidal cell dendrites has been reported (Wong et al., 1979; Benardo et al., 1982). Perhaps this sink represents the activation of a portion of dendritic membrane by the postsynaptic potential; activation of such channels may play a role in bringing pyramidal cells to a discharge threshold. This sink in the proximal apical dendrites persists long after the population spike has terminated. If the sink were attributable to activation of calcium channels, the slow kinetics of calcium channel inactivation may help explain its long-term nature, and also account for the common occurrence of depolarizing afterpotentials in pyramidal cells (Kandel and Spencer, 1961). Previous CSD studies in CA1 (Leung, 1978; Cauller et al., 1985) or in dentate (Jefferys, 1979) have not reported the presence of a sink in the proximal apical dendrites. However, the spatial sampling intervals used in those studies were much larger than our sampling interval of $30 \mu \mathrm{m}$. Since the magnitude of this early sink was large only along the radiatum/pyramidale border, it may have easily been missed.

The findings from the CSD experiments following development of LTP are inconclusive. The results from the experiments with the triple-electrode array, comparing potentiated responses at low stimulus intensity with pre-HFS responses at higher stimulus intensity, indicated a small tendency for the early sink in radiatum/pyramidale to shift distally following induction of LTP. The results averaged across all 9 experiments show this trend. However, later experiments that employed a single moving electrode to calculate CSDs did not show a shift in the early sink. In theory, there should be no difference between the 2 procedures. We compared methods for CSD analysis within a given experiment; CSDs computed using the field potential averages from the middle electrode of the triple-electrode array were not significantly different from the CSDs based on the single- and triple-electrode procedures. The discrepancy between procedures also cannot be attributed to misplacement of the tripleelectrode array: when the post-HFS track of extracellular potentials is compared to the pre-HFS measures, the pattern of sinks and sources at early and late time points (i.e., before and after the sink shift time points $c$ and d) superimpose (i.e., they align at identical locations along the neuron, see time points a and $f$ in Figs. 3 and 4). Finally, shifting of the sink following HFS was not a function of the degree of potentiation in the population EPSP or population spike.

The roles that the early sink may serve in cell function and development of LTP are currently unclear. However, recent studies offer possible insights. Kelso et al. (1986) reported that LTP is induced in a weak afferent input (i.e., where HFS of the orthodromic pathway alone will not induce LTP) when the HFS of the afferent input is paired with intracellular injection of depolarizing current. This finding suggests that there are depolarizing current-activated processes in the membrane (possibly dendritic) that facilitate the induction of LTP. It is possible that depolarizing current activates excitatory channels (e.g., NMDA-activated cation channels) in the proximal portion of the apical dendrites, which contribute to the early sink seen in our experiments. Wigstrom and Gustafsson (1984) showed that induction of LTP only occurred when there was a negative extracellular voltage shift in the dendrites during the HFS period; they interpreted this voltage shift as a sign of a dendritic depolarizing process that may be involved in mediating LTP. This extracellular negativity may correspond to activation of the membrane channels contributing to the early sink.

It is also interesting to speculate about the possible significance of a post-HFS shift in the carly sink. Movement of the sink with development of LTP might indicate the creation of a 


\section{Comparison of CSD Amplitudes for Orthodromic and Antidromic Stimulation}
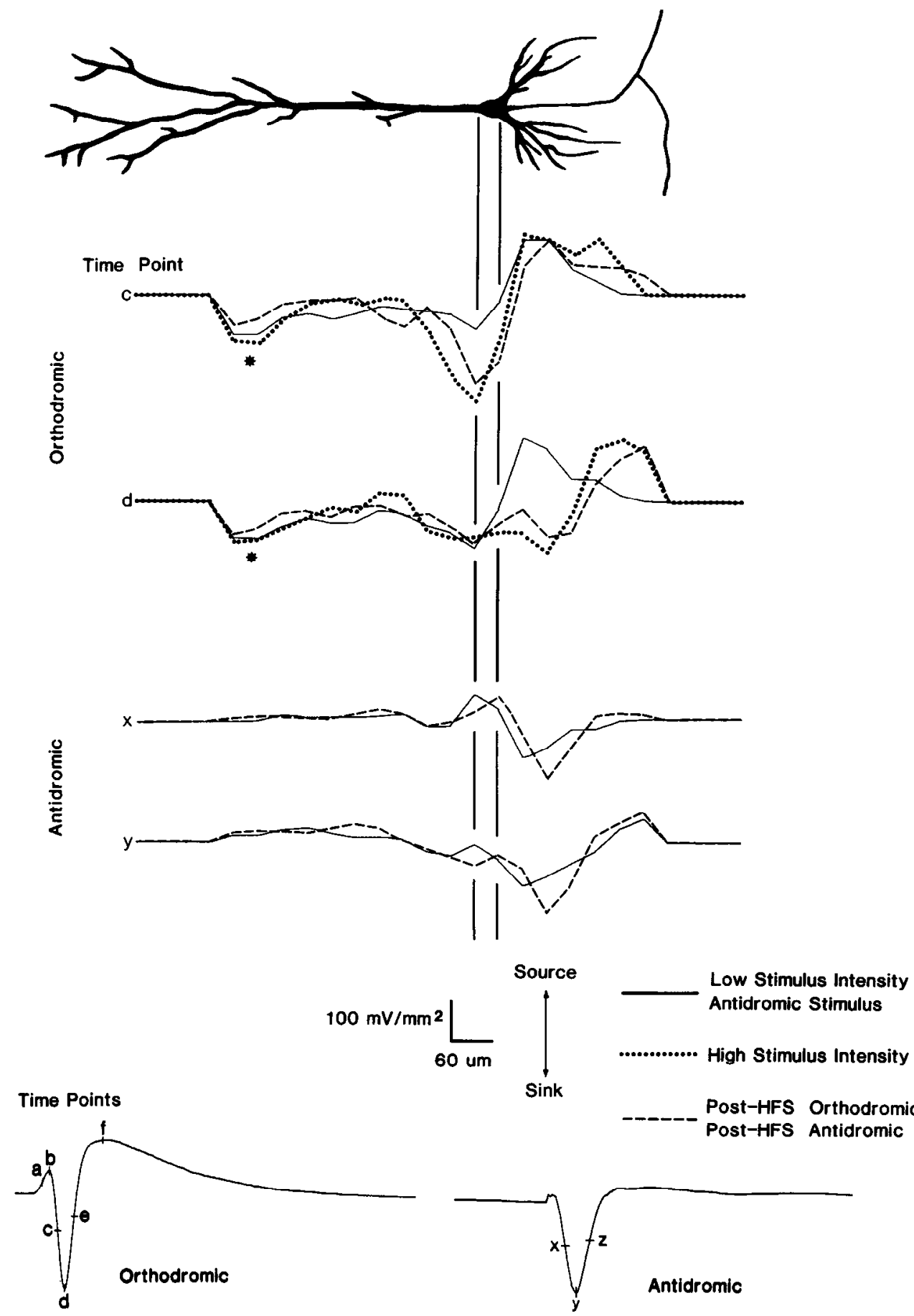

Figure 6. Comparison of CSD measures for orthodromic and antidromic stimulation. Time points $\mathrm{c}$ and $\mathrm{d}$ were selected from orthodromically evoked waveforms recorded in str. pyramidale (see inset at lower left); time points $\mathrm{x}$ and $y$ were selected from antidromically evoked waveforms (see inset at lower right). The "second-nearestneighbor technique," using an interelectrode distance of $60 \mu \mathrm{m}$, was used to compute the CSDs (see text for details). CSD amplitudes (ordinate) are shown relative to electrode position along the pyramidal neuron axis $(a b$ scissa). An apical dendritic sink, associated with synaptic activation, is seen with orthodromic stimulation (asterisks in traces $\mathrm{c}$ and $\mathrm{d}$ ); this "synaptic" sink was not detectable when smaller spacing distances (i.e., $30 \mu \mathrm{m}$ ) were used to compute the CSDs (see Figs. 3 and 4). The early sink and source are present during orthodromic stimulation (traces $c$ and d) but not during antidromic stimulation (traces $x$ and $y$ ). The late sink and source are present under both stimulating conditions. With orthodromic stimulation, there was no postHFS shift of either early or late sink. new active zone along the dendritic membrane. A shift of the sink in a distal direction suggests that the new active zone is closer to the site of EPSP generation - and thus more likely to be activated by the EPSP. This active portion of dendritic membrane may then play a role in bringing cells to discharge threshold; the increased likelihood of activation of this dendritic segment would help explain the increased probability of cell discharge during LTP. The movement of such an active zone would have no necessary effect on EPSP potentiation, and it could explain the dissociation between EPSP and spike potentiation in LTP.

At least 2 difficult objections can be raised to the notion that a shift of the early sink contributes to E-S potentiation. First, LTP in the CAl area has been shown to be specific to the tetanized pathway (Schwartzkroin and Wcstcr, 1975; Andersen et al., 1977, 1980; Alger et al., 1978). It is difficult to explain 


\section{A}

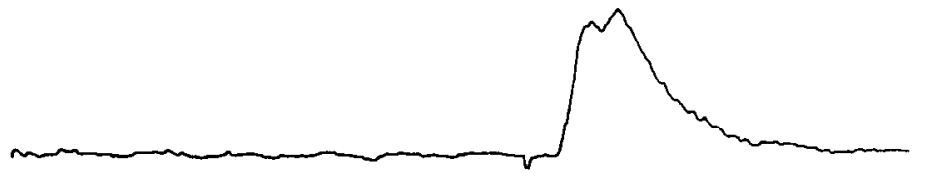

S1

Figure 7. Paired-pulse paradigm for determining independence of inputs in the double orthodromic stimulation experiments. To insure that the 2 stimulating electrodes in str. radiatum activated different groups of fibers, stimulating electrodes were positioned such that a test pulse delivered to S1 showed no facilitation when preceded by a conditioning pulse delivered to $S 2$. $A$, Response to stimulation of $\mathrm{S} 1$ alone. $B$, Heterosynaptic paired-pulse condition. The test pulse to $S 1$ preceded (50 $\mathrm{msec}$ ) by a conditioning pulse at $\mathbf{S} 2$. The response to SI stimulation shows no population spike facilitation. Facilitation would be expected if S1 and S2 were activating an overlapping set of fibers. $C$, Homosynaptic paired-pulse condition. The test pulse to $S 1$ was preceded $(50 \mathrm{msec})$ by a conditioning pulse to the same electrode. The response to the second stimulus of the pair shows dramatic facilitation of the population spike.
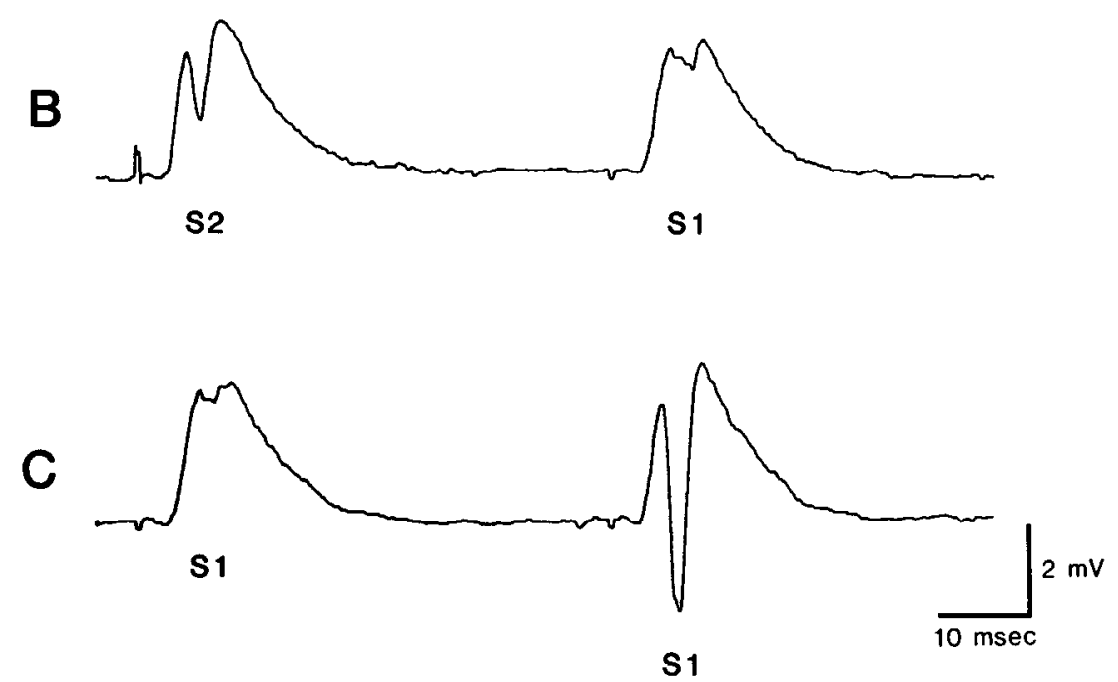

how a change in the proximal dendrites (close to the cell body) would maintain such specificity; activated synapses in any part of the apical dendritic tree might excite this portion of dendritic membrane. A change in location of this proximal dendritic sink should have an impact on the ability of EPSPs, generated almost anywhere in the apical dendrites, to bring the cell to discharge threshold. Interestingly, there is now some indication that E-S (as opposed to the V-E) potentiation in the CAl is actually heterosynaptic, as shown by Abraham et al. (1985) for the dentate. Another objection to the hypothesis that sink movement contributes to LTP concerns our finding that no alteration in spike discharge threshold was measured in our intradendritic recordings following development of LTP (Taube and Schwartzkroin, 1988). Since our intradendritic recordings were distal to the portion of dendritic membrane associated with the early sink, an apical shift in the sink would be expected to influence the amount of injected current (in the dendrite) required to discharge the cell. Indeed, it remains unclear if sink movement does occur following HFS, or how it might explain the E-S component of LTP.

In conclusion, the results from the CSD experiments indicate that 2 sinks develop near the cell body layer (in addition to the sink associated with the EPSP) in response to orthodromic stimulation of the apical dendrites. The early sink is (1) present along the radiatum/pyramidale border, (2) evident throughout the time course of the evoked field potential, (3) peaks in magnitude just prior to the peak of the population spike, and (4) is associated only with orthodromic stimulation. The second, or late, sink occurs in the proximal portion of the basal dendrites and has a characteristic time course similar to the population spike. In some experiments, the early dendritic sink shifted apically following development of LTP; however, this change was inconsistent, and of questionable functional significance.

\section{References}

Abraham, W. C., T. V. P. Bliss, and G. V. Goddard (1985) Heterosynaptic changes accompanying long-term but not short-term potentiation of the perforant path in the anaesthetized rat. J. Physiol. (Lond.) 363: 335-349.

Alger, B. E., A. L. Megela, and T. J. Teyler (1978) Transient heterosynaptic depression in the hippocampal slice. Brain Res. Bull. 3: 181184.

Andersen, P., S. II. Sundberg, O. Sveen, and H. Wigstrom (1977) Specific long-lasting potentiation of synaptic transmission in hippocampal slices. Nature 266: 736-737.

Andersen, P., S. H. Sundberg, O. Sveen, J. W. Swann, and H. Wigstrom (1980) Possible mechanisms for long-lasting potentiation of synaptic transmission in hippocampal slices from guinea pig. J. Physiol. (Lond.) 302: $463-482$.

Andersen, P., M. Avoli, and O. Hvalby (1984) Evidence for both preand postsynaptic mechanisms during long-term potentiation in hippocampal slices. Exp. Brain Res. Suppl. 9: 315-324.

Benardo, L. S., L. M. Masukawa, and D. A. Prince (1982) Electrophysiology of isolated hippocampal pyramidal dendrites. J. Neurosci. 2: 1614-1622.

Bliss, T. V. P., and T. Lomo (1973) Long-lasting potentiation of synaptic transmission in the dentate area of the anaesthetized rabbit following stimulation of the perforant path. J. Physiol. (Lond.) 232: 331-356.

Cauller, L. J., N. Chiaia, and T. J. Teyler (1985) The neural basis of evoked field potentials studied by current-source density (CSD) analysis of the hippocampal slice as a simple model system. Soc. Neurosci. Abstr. 11: 505 .

Creager, R., T. Dunwiddie, and G. Lynch (1980) Paired-pulse and frequency facilitation in the CA1 region of the in-vitro rat hippocampus. J. Physiol. (Lond.) 299: 409-424.

Dietzel, I., U. Heinemann, G. Hofmeier, and H. D. Lux (1982) Changes in the extracellular volume in the cerebral cortex of cats in relation to stimulus induced epileptiform afterdischarges. In Physiology and Pharmacology of Epileptogenic Phenomena, M. R. Klee, H. D. Lux, and E. J. Speckmann, eds., pp. 5-12, Raven Press, Ncw York.

Freeman, J. A., and C. Nicholson (1975) Experimental optimization 
of current-source density technique for anuran cerebellum. J. Neurophysiol. 38: 369-382.

Jefferys, J. G. R. (1979) Initiation and spread of action potentials in granule cells maintained in-vitro in slices of guinea pig hippocampus. J. Physiol. (Lond.) 289: 375-388.

Jefferys, J. G. R. (1984) Current flow through hippocampal slices. Soc. Neurosci. Abstr. 10: 1074.

Kandel, E. R., and W. A. Spencer (1961) Electrophysiology of hippocampal neurons. II. After-potentials and repetitive firing. J. Neurophysiol. 24: 243-259.

Kelso, S. R., A. H. Ganong, and T. H. Brown (1986) Hebbian synapses in hippocampus. Proc. Natl. Acad. Sci. USA 83: 5326-5330.

Leung, L. S. (1978) Hippocampal CAl region-Demonstration of antidromic dendritic spike and dendritic inhibition. Brain Res. 158: 219-222.

McNaughton, B. L., and C. A. Barnes (1977) Physiological identification and analysis of dentate granule cell responses to stimulation of the medial and lateral perforant pathways in the rat. J. Comp. Neurol. 175: 439-454.

Nicholson, C., and I. A. Freeman (1975) Theory of current sourcedensity analysis and determination of conductivity tensor for anuran cerebellum. J. Neurophysiol. 38: 356-368.

Ranck, J. B., Jr. (1963) Specific impedance of rabbit cerebral cortex. Exp. Neurol. 7: 144-153.
Schwartzkroin, P. A., and K. Wester (1975) Long-lasting facilitation of a synaptic potential following tetanization in the in vitro hippocampal slice. Brain Res. 89: 107-1 19.

Taube, J. S., and P. A. Schwartzkroin (1985) A current-source density analysis of hippocampal CAl region following LTP. Soc. Neurosci. Abstr. 11: 778

Taube, J. S., and P. A. Schwartzkroin (1988) Mechanisms of longterm potentiation: EPSP/spike dissociation, intradendritic recordings, and glutamate sensitivity. J. Neurosci. 8: 1632-1644.

Wigstrom, H., and B. Gustafsson (1984) A possible correlate of the postsynaptic condition for long-lasting potentiation in the guinea pig hippocampus in vitro. Neurosci. Lett. 44: 327-332.

Wilson, R. C. (1981) Changes in translation of synaptic excitation to dentate granule cell discharge accompanying long-term potentiation. I. Differences between normal and reinnervated dentate gyrus. J. Neurophysiol. 46: 324-338.

Wilson, R. C., W. B. Levy, and O. Steward (1981) Changes in translation of synaptic excitation to dentate granule cell discharge accompanying long-term potentiation. II. An evaluation of mechanisms utilizing dentate gyrus dually innervated by surviving ipsilateral and sprouted cross temporodentate inputs. J. Neurophysiol. 46: 339-355.

Wong, R. K. S., D. A. Prince, and A. I. Basbaum (1979) Intradendritic recordings from hippocampal neurons. Proc. Natl. Acad. Sci. USA 76: $986-990$. 